\title{
Automatic non-destructive quality inspection system for oil palm fruits
}

\author{
Muhammad Makky ${ }^{1,2,3}$, Peeyush Soni ${ }^{1 *}$, and Vilas M. Salokhe ${ }^{4}$ \\ ${ }^{1}$ Agricultural Systems and Engineering, Asian Institute of Technology, Pathumthani 12120, Thailand \\ ${ }^{2}$ Department of Agricultural Engineering, Andalas University, West Sumatera 25163, Indonesia \\ ${ }^{3}$ School of Agricultural Technology, Alexander Technological Educational Institute of Thessaloniki, Thessaloniki 57400, Greece \\ ${ }^{4}$ Kaziranga University, Jorhat, Assam, India
}

Received March 11, 2013; accepted November 18, 2013

\begin{abstract}
A b s t r a c t. In this research a non-destructive, rapid and cost effective examination machine for the estimation of the ripeness fraction, oil content and free fatty acid level in oil palm fresh fruits bunch was developed. The automatic machine-vision based inspection system provided consistency, rapid estimation and acceptable accuracy results in non-destructive manner. Fresh fruits bunch samples from Tenera cultivar ( 7 to 20 years trees) were taken from Cimulang plantation, Bogor, Indonesia. Two statistical analysis methods were used: a forward stepwise multiple linear regression analysis and a multilayer-perceptron artificial neural network analysis. The best prediction of ripeness and oil content models were obtained using the latter method, while the best free fatty acid prediction model was developed by the first method. The models were then employed in the machine-vision inspection systems of the machine. The system best prediction accuracy of ripeness, oil content and free fatty acid models was $93.5,96.41$, and $89.32 \%$, with standard error of prediction being $0.065,0.044$ and 0.068 , respectively. The system was tested through a series of field tests, and successfully examined more than $12 \mathrm{t}$ of fruits bunch per hour, without causing damage.

K e y w o r d s: oil palm, fresh fruits bunch, automatic quality inspection, machine vision, non-destructive evaluation
\end{abstract}

\section{INTRODUCTION}

The quality of oil palm (Elaeisguineensis Jacq.) fresh fruit bunches $(F F B)$ can be determined by their condition $e g$. ripeness, damage or bruises. Correspondingly, the purchasing value of FFBS should be based on bunch appearance and prediction of the quantity and quality of oil that can be extracted from the bunch. Ripe bunches are desirable (Fig. 1), since they have more oil compared to unripe ones, with lower free fatty acid $(F F A)$ than over-ripe bunches (Siregar, 1976). This makes the bunch ripeness become a major factor for determining the quantity and quality of oil yield. Ideally, upon purchasing the $F F B$, oil palm mills should know the oil contains of each $F F B$ as well as its quality (Makky et al., 2013a). However, only ripeness can be determined by manual visual inspection, while other properties cannot be determined until the milling process is completed (Makky and Soni, 2014). Although FFBs oil content (OC) can be precisely measured manually in the laboratory, it is neither cost effective nor feasible to analyse every single $F F B$ in the processing line. Another constraint in $F F B$ manual quality inspection is the risk of damage and injury to FFBs during inspection, which should be avoided, since it will reduce the quality of oil due to increment of FFA level in the fruits caused by acceleration of lipolytic activity (Hadi et al., 2009). Level of $F F A$ determines the price and quality of crude palm oil (CPO) produced, and affects the decision on method of production, storing and marketing of the CPO (Saad et al., 2006). To address these problems, a non-destructive, rapid and cost effective examination system that can determine ripeness and estimate the $O C$ and $F F A$ level in every $F F B$ is required. An automatic inspection machine with machinevision-based system may provide consistent and quick examination with acceptable accuracy and non-destructive nature.

A wide range of applications of imaging techniques for agricultural products have been used to assess the physical and chemical properties of commercial agricultural products such as apples (Nicolai et al., 2007; Zdunek et al., 2014), barley (Zapotoczny, 2012), beets (Arenas-Ocampo et al., 2012), date fruits (Mireei et al., 2010), eggs (Asadi et al., 2012), maize seeds (Hernandez et al., 2011), rose peduncles (Matsushima et al., 2013), wheat (Arefiet al., 2011), as well as other biological material (Briseńo-Tepepa et al., 2008; Gonzalez-Ballesteros et al., 2006). 


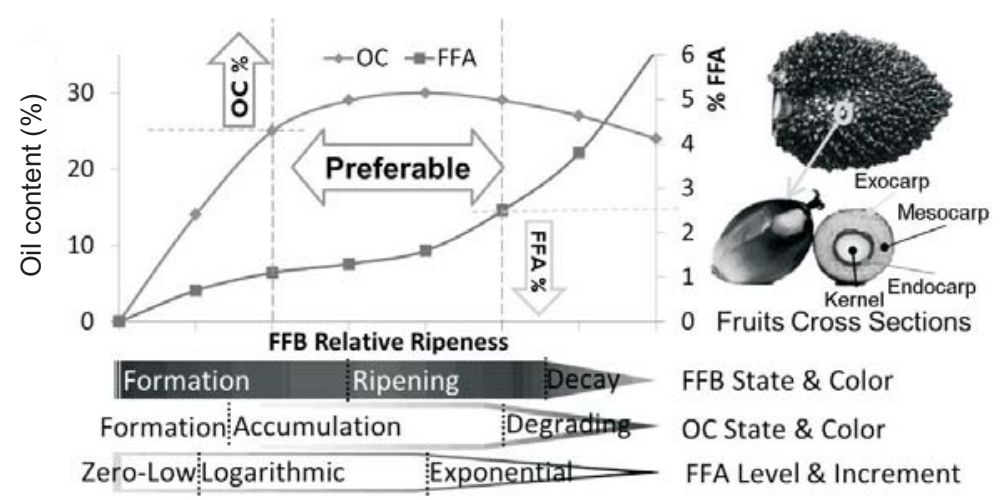

Fig. 1. Physical and chemical development of $F F B$ upon maturing.

In addition, the application of imaging technologies in an automatic inspection system for commercial agricultural products increases the machines potency in terms of speed, volume and continuity of work (Hernandez et al., 2010; Koenderinket al., 2010; Kondo, 2010).

Using machine vision, several researches regarding $F F B$ ripeness assessment have been carried out (Jaffar et al., 2009; Jamil et al., 2009; Makky et al., 2004; Makky and Soni, 2013b; Roseleena et al., 2011). However, those works were focused mainly on ripeness determination of $F F B$ based on its physical appearance. On the other hand, only limited literature is available on the relationship between physical appearance of $F F B$ and its oil content (Razali et al., 2011; Shaaraniet et al., 2010), as well as association of physical appearance of $F F B$ with its FFA (Junkwon et al., 2009; Makky and Soni, 2014). Nonetheless, all those studies were undertaken either under laboratory conditions or without the use of machine vision or automation technology. The estimation of $F F B S$ oil content and $F F A$ still remains a manual task which is time consuming, expensive and labour intensive.

The objective of this work was to assess the quality parameters of oil palm $F F B$ by utilising machine-vision based automatic inspection system platform. In the previous work (Makky and Soni, 2013b), an automatic grading machine for oil palm $F F B$ was developed with the main focus on the determination of physical appearance quality of the $F F B$. In this research, further development of this machine was done, that enables the machine to assess not only the ripeness of the $F F B$, but also its $O C$ and $F F A$ properties by means of non-destructive technique. The performance of the inspection machine was compared to manual and laboratory assessment for these three features. The tests were carried out under field operation.

\section{MATERIAL AND METHODS}

For the assessment purpose, the inspection machine from previous work (Makky and Soni, 2013b) was upgraded, including its mechanical system, control system, and machine vision system. The new system comprized mechanical platform, electronic control systems, and operational systems.
The mechanical platform included a ladder type chas- sis to support the whole equipment, evenly-arranged idle rollers to support the conveyor belt, dynamic rollers driven by the electric motor through speed reducer gearbox and chainsprocket power transmissions to rotate the conveyor, and a separator gate driven by two motors and limit switches to segregate the substandard fruits bunch. The electronic control systems consisted of a portable computer as central processing unit, single chip programmable interface for controlling moving parts and actuators, and input-output analogue-to-digital and digital-to-analogue peripherals for interfacing the mechanical parts with the computer, thus enabling communication and feedback between components and control systems. The whole system runs by an operational system in the form of a custom made image processing program, performing the qualitative and quantitative ananlyses of the samples.

Using the same chassis platform, modification was done in several sections of the machine. The conveyor belt was repainted with white colour to provide best contrast between the belt and the object $(F F B)$, which enables image to be segmented properly. The tone surface of conveyor enables light to be scattered, thus reducing reflection of light that might interfere during image acquisitions. The power drive and transmission unit were unchanged, while the dampers were replaced to provide more stable (shock-free) conveyor belt operation. The separator movement is now limited to only $45^{\circ}$ to ease segregation of poor quality $F F B$, according to inspection results.

The control system layout was revised accordingly. The position of the laser emitter and light dependent resistors (LDR) sensor were placed further back down toward the exit of the inspection chamber (Fig. 2). The repositioning was aimed to enable the whole $F F B$ section to be captured in the image, especially for the extremely large bunch. The software program for running the single chip microcontroller (SCM) was modified to accommodate the repositioning of LDR sensor as well as the adjustment of limit switches that govern separator gate movement, while maintaining the In System Programmable (ISP) capability. 
The real time image acquisition and analysis was improved. The image processing software in machine vision system was modified to enable assessment of two other $F F B$ quality properties, ie $O C$ and $F F A$ (Fig. 3). The image processing software was developed using C\# (SharpDevelop 3.2, IC\#Code Team) and Visual Basic (VB 6.0, Microsoft)

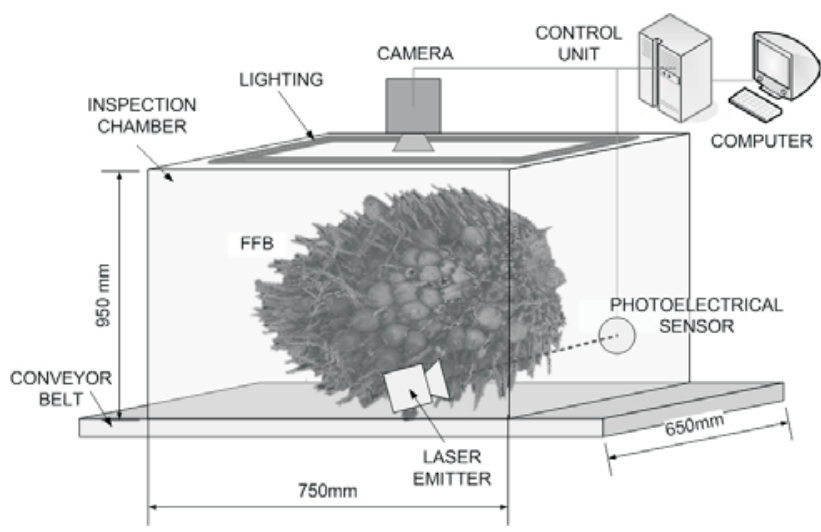

Fig. 2. Reposition of laser detection in the machine vision inspection system. programming by utilizing native Win32 application programming interface (API). The captured $F F B$ image was segmented to extract the features, in order to calculate the ripeness, $O C$ and $F F A$. The separation of $F F B$ is based on its ripeness, $O C$ and FFA estimation by the software (Fig. 4). The whole system runs automatically without the necessity of an operator to control the process. However, one person labour was required to feed the $F F B$ onto the conveyor belt. The inspection chamber dimensions and conveyor belt speed, as well as the procedure of feeding FFB onto the machine, ensure that only one $F F B$ enters the chamber at a time.

The FFB features from each captured image were calculated and stored. The features included the number of Pixels in the object as well as its red-green-blue (RGB) digital number (DN). The data of each pixel were calculated and stored in variables, namely object pixel, R, G, and B. The performance of colour recognition using machine vision system relies heavily upon the choice of appropriate colour range and the characteristics of variables employed in the calibration model. Therefore, in this work, the image processing program transforms image RGB colour model into hue-saturation-intensity (HSI) (Gonzalez and Woods, 2008) to generate more information on the object. The results were

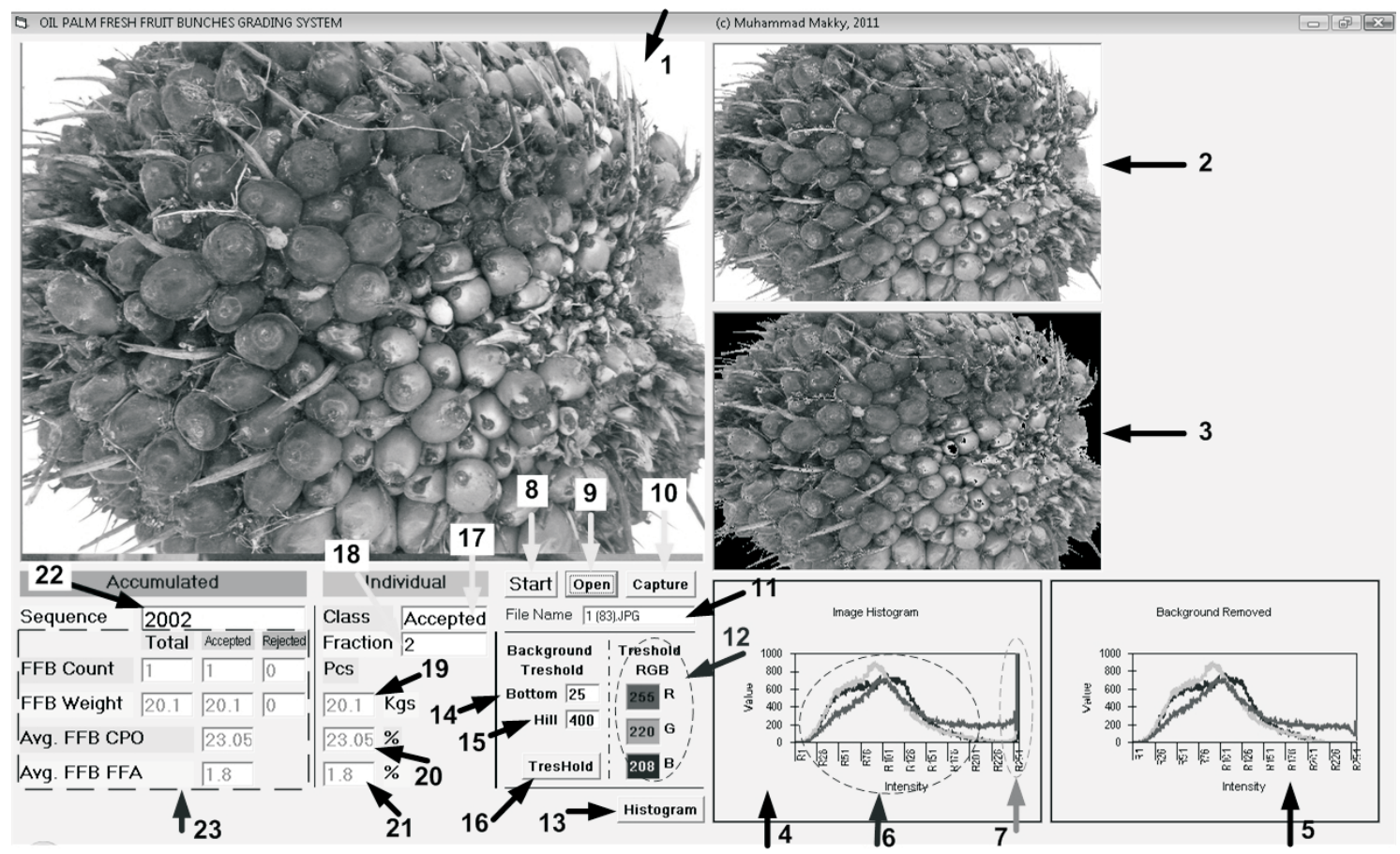

Fig. 3. The image processing program display. 1. Camera live view, 2. Snapshot image, 3. Segmented image, 4. RGB histogram from recorded image, 5. RGB histogram from segmented image, 6. Object of interest, 7. RGB background, 8. Run system with Single Chip Microcontroller SCM interfacing I/O operation, 9. Run program using stored image, 10. Run system by manually capturing frame from camera, 11. Uploaded file name, 12. RGB threshold value for segmentation, 13. Reload segmented image histogram after manually changed threshold value, 14. Initial lower bound for adaptive thresholding, 15. Initial upper bound for adaptive thresholding, 16. Reload the threshold after manually changed bottom and hill value, 17. Current $F F B$ classification result accepted $=\mathrm{F} 1, \mathrm{~F} 2, \mathrm{~F} 3$; rejected $=\mathrm{F} 00$, F0, F4, F5, F6., 18. Current $F F B$ Fraction, 19. Current $F F B$ mass estimation, 20. Current $F F B O C$ estimation, 21. Current $F F B ~ F F A$ estimation, 22. Current $F F B$ sequence, 23. Total $F F B$ inspected, and the average of mass, $O C$ and $F F A$. 
assigned as variables $\mathrm{H}, \mathrm{S}$, and I. To minimize the effect of change in intensity, normalization of RGB DN data into normalized: R (r), G (g) and B (b) were performed by methods mentioned in Makky and Soni (2013b). Another feature extracted from the image was ripeness index (RI) described by Roseleena et al. (2011). That set of variables (object pixel, R, G, B, H, S, I, r, g, b, RI) was used as independent variables for generating models for the estimation of $F F B S$ ripeness, oil content and $F F A$.

The $F F B$ scan be classified into 8 ripeness fractions (Makky and Soni, 2013b). In this research, 180 FFB samples of Tenera cultivar were taken randomly from oil palm trees aged 7 to 20 years, from Cimulang plantation, Bogor, Indo-

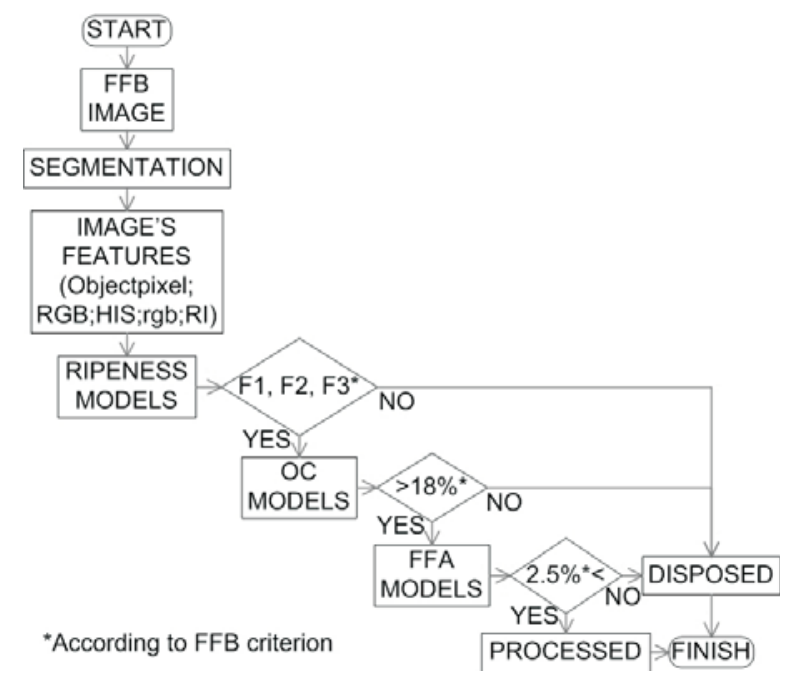

Fig. 4. $F F B$ hierarchy grading selection. nesia. All FFB images were captured in the inspection chamber to extract the features from the object. To inactivate the lipases, the samples were boiled immediately. Oil that dissolved into water during boiling was measured using the method described by American Public Health Association (APHA, 2005).

For assessing the ripeness of each $F F B$ sample, a panel of three experienced graders was appointed. In the laboratory analysis, 25 samples of fruitlets from each bunch were used to analyse the oil content and FFA level in the laboratory. The fruit samples were taken from different sections of each bunch ie. top, middle, and bottom part. The number of fruits selected from the inner, middle, and outer part of the bunch (Fig. 5) were eight, eight and nine fruits, respectively. The tests were conducted during November until December 2011.

For measuring the oil content in fruit mesocarp, fruit samples were detached from spikelet, and pared to separate the mesocarp. The mesocarp was then weighed using analytical balances (Sartorius, BP 160 P, Germany) for ensuring high measurement accuracy. Samples were then dried in the oven for one day to remove physical water from the mesocarp. The oil in the mesocarp was extracted using a soxhlet extractor, with hexane as chemical solvent. The extraction process was complete after hexane colour in the soxhlet became translucent. The remaining fibre and the oil solution in the thimble were dried to remove the dissolved hexane, and then cooled in a desiccator. It was then weighed in the analytical balance, and the result was recorded for mesocarp oil content calculation, as specified by Indonesian Oil Palm Research Institute (IOPRI, 1997). The mesocarp oil (Oil $\left.{ }_{m}\right)$ can be calculated as:

$$
\% \mathrm{Oil}_{m}=\frac{M_{1}-M_{2}}{M_{3}} 100 \%
$$
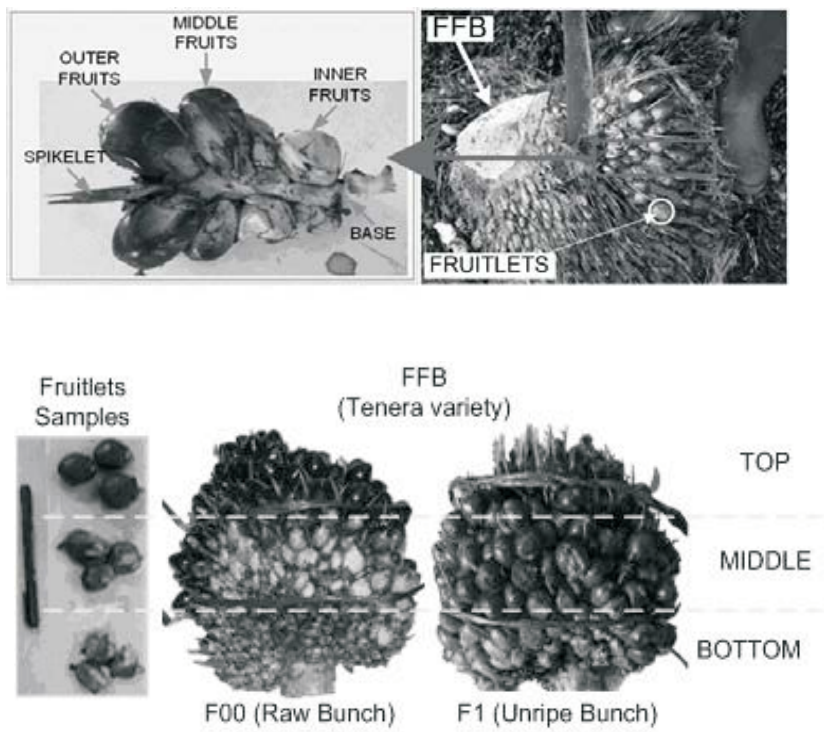

Fig. 5. Fruitlets samples from bunch taken from top, middle, and bottom section ( 8 inner fruits, 8 middle fruits, and 9 outer fruits). 
where: $M_{1}$ is thimble and oil mass $(\mathrm{g}), M_{2}$ is empty thimble mass $(\mathrm{g})$, and $M_{3}$ is mesocarp sample mass $(\mathrm{g})$.

The CPO recovery from the sample $F F B$ was calculated using the equation:

$$
\% O C=\frac{\sum M_{f}}{M_{F F B}} \% M_{m} \% O i l_{m}
$$

where: $O C$ is oil content, $M_{f}$ is fruitlets mass $(\mathrm{kg}), M_{F F B}$ is mass of $F F B(\mathrm{~kg}), \% M_{m}$ is percentage of mesocarp mass from fruitlets $(\%), \% O i l_{m}$ is percentage of mesocarp oil.

Free fatty acid is considered as an important quality parameter for CPO. FFA is formed in the process of oil hydrolysis to become acid. Accelerated by light and heat, FFA formed in CPO decreases the smoking point (Bobbio and Bobbio, 2001) and is responsible for undesirable flavours and aroma (Osawa et al., 2007).

Since the oil extraction process using the soxhlet involves heat and requires significant time, there might be a risk that the extracted oil becomes degraded and raises the FFA level, and thus significantly affects the measurement accuracy. For this reason, the oil used for $F F A$ level assessment in this work was taken from the mesocarp of boiled fruits samples, and extracted using a mechanical squeezer. This was done to evade oil degradation and to increase the accuracy of FFA measurement. The FFA in the oil was measured by the titration method (IOPRI, 1997; AOCS, 2004; NSAI, 2006). The percentage of $F F A$ in CPO was calculated as palmitic acid and interpreted as the mass of $\mathrm{KOH}$ (in milligrams) required to counteract acid from $1 \mathrm{~g}$ of sample. In this research, $F F A$ was measured using the procedure defined by IOPRI (1997) in accordance with standards established by the National Standardization Body of Indonesia, SNI 01-2901-2006 (NSAI, 2006). This procedure meets the qualification according to the American Oil Chemist Society (AOCS, 2004) official method Ca 5a-40. The percentage of $F F A$ (as palmitic acid) is expressed as:

$$
\% F F A(\text { as palmitic })=\frac{25.6 N V}{M},
$$

where: $V$ - volume of $\mathrm{KOH}(\mathrm{ml}), N$ - normality of $\mathrm{KOH}$, and 25.6 is the constant based on the molar mass of palmitic acid, and $M$ - sample mass (g).

For modelling the ripeness, $O C$, and $F F A$ of oil palm $F F B$ in the image processing algorithm, two analyses were performed using Forward Stepwise Multiple Linear Regression (FS-MLR) and Multilayer-Perceptron Artificial Neural Network (MP-ANN) method. These two methods were introduced using Statistical engineering software (IBM, USA) to generate the $F F B$ quality models: ripeness fraction, $O C$ and $F F A$ prediction. The models are created by regressing manual assessment results with features extracted from the images (ie. Object pixel, R, G, B, H, S, I, r, g, b, RI). Insignificant variables were identified and removed using F-statistics. Data acquired from 180 samples were split evenly into three parts. The first two parts were used as data training for creating the models, and the rest was used for cross-validation of the accuracy of the models.
The FS-MLR analysis for modelling has been successfully used for model calibration (Liu et al., 2008; Makky and Soni, 2014; Naes and Mevik, 2001). This method can improve model accuracy by removing collinearity in variables, thus increasing the efficiency of the algorithm results. By introducing fewer variables compared to common Multi Linear Regression analysis, the prediction accuracy of the model generated using this method can be expected to increase significantly. The FS-MLR prediction models were constituted of several variables, however, it is precarious to determine the importance of each variable due to collinearity problems, although the exegeses of the model remain possible. The advantage of models generated using FS-MLR method is the simplicity of the equation to be written in mathematical form. Common multiple linear regression can be written as:

$$
y_{i}=\beta_{0}+\beta_{1} x_{1}+\ldots+\beta_{n} x_{i}+e_{i}
$$

where: $y_{i}$ is regression, $x_{i}$ is input variables or predictors, $\beta_{I}$ is regression coefficients, $\beta_{0}$ is intercept and $e_{i}$ is error term.

The MP-ANN analyses for modelling the $F F B$ quality assessments were used for approximation estimation of $F F B$ qualities (ie. ripeness, $O C$ and $F F A$ ) by training a set of multilayer perceptron, input layers, hidden layers and output layer. To avoid over-fitting of the models upon training, which may lead to poor performance upon validation, the set of hidden layers and processing elements were set to be changed automatically by the software.

All models were built using statistical engineering software (IBM, USA). In analysing the data, confidence level of 99.99 was used $(p<0.01)$, along with boosting accuracy option in the software.

The models performance was evaluated by comparing the prediction results and measured values, in the validation sets. The main performance statistics for model validation were the coefficient of determination $\left(\mathrm{R}^{2}\right)$, the standard error of calibration (SEC) and the standard error of prediction (SEP) (Makky and Soni, 2013b; 2014).

\section{RESULTS AND DISCUSSION}

The inspection machine was run through a series of tests in actual field conditions. During the tests, the accuracy of the models was validated. The effectiveness of operation was examined for all components of the developed machine. Feeding of $F F B$ by the operator resulted in irregular orientation of the bunch on the conveyor belt. However, the results show no significant differences.

Chemical analysis of the $F F B$ sample was done to measure actual $O C$ and $F F A$ in each bunch. During the ripening process, $F F B$ oil content as well as its $F F A$ increase. Samples selected for this research had different ripeness levels. Based on their ripeness (fraction), the category-wise $O C$ and FFA results are presented in Fig. 6. Most of the FFB 
a

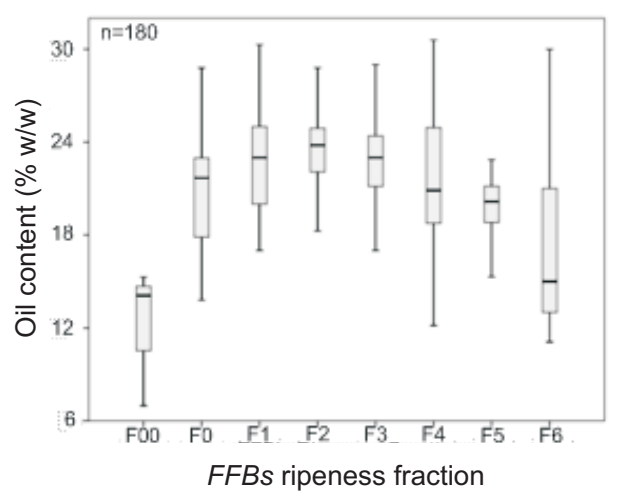

b

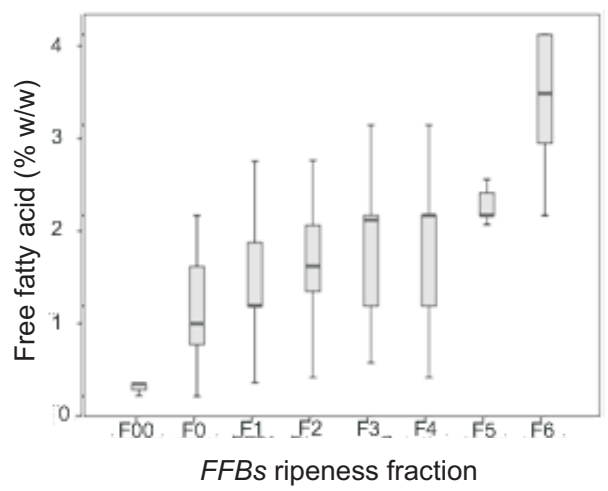

Fig. 6. Measured: $\mathrm{a}-O C$, and $\mathrm{b}-F F A$ according to ripeness fraction.

$\mathrm{a}$

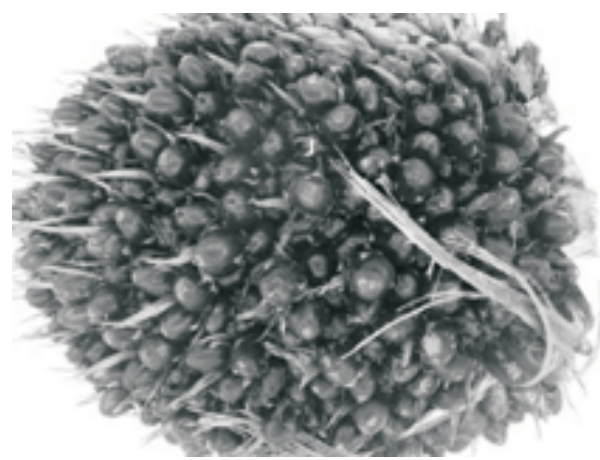

$\mathrm{b}$

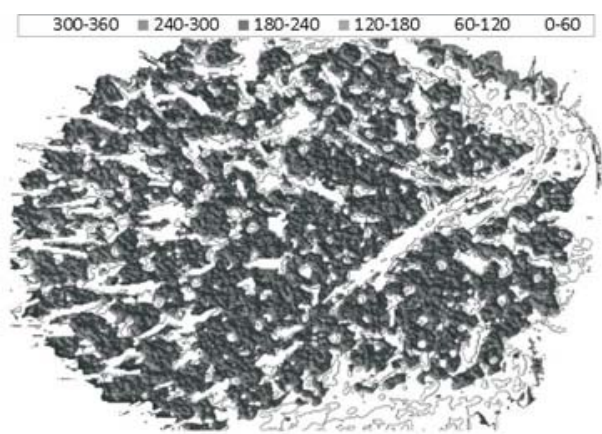

Fig. 7. Texture analysis of $F F B$ image: a - original image, $\mathrm{b}$ - textured image.

samples of fractions 4,5 and 6 had lower $O C$ compared to $F F B$ in fractions 1, 2, and 3, while the FFA level was vice versa. The reason was, when calculated as the whole bunch, the percentage of oil content in $F F B$ was decreased due to the increasing number of detached fruitlets in the field, many of which cannot be recovered due to various factors. The desirable $F F B$ ought to have a high $O C$, while the $F F A$ in the oil is low. The chemical analysis results showed that moderately ripe $F F B S$ within fraction range of 1 to 3 are preferred over the higher fractions, considering their $O C$ and $F F A$.

Images captured by the camera were segmented by the image processing program to remove the background. The adaptive thresholding algorithm used in the program separated the object from its background in the image, rapidly and accurately, without losing object features. A texture analysis of the image was done to classify the RGB value of specific components of the object in order to distinguish among fruitlets, spikelets, and other parts (Fig. 7).

The case of multiple linear regressions in real-world regression models involves multiple predictors, where the response variable y or regression is still a scalar. Therefore, the model for ripeness fraction of oil palm $F F B$ can be written as:
Fraction $=-4.110^{-5}$ Object pixel $+8.4310^{-2} R+6.9510^{-2} G$ $-6.0310^{-2} B+14.39 g-5.05 b+0.012 H-9.0610^{-2} I-1.81$ (5)

where: Fraction is FFB ripeness fraction estimation, Object pixel is the number of pixel in the object, R, G, and B are the average of red, green, and blue $\mathrm{DN}$ in the object, $g$ and $b$ are the average of normalized green and blue DN in the object, $H$ and $I$ are the average of hue and intensity DN in the object. In fraction model, two predictors are excluded, $r$ and $S$, for being insignificant $(\mathrm{p}>0.01)$.

As for the model of oil content $(O C)$, it can be written as:

$O C=8.310^{-2} R-9.8610^{-2} B-17.82 r+51 g-51.89 b+$

$0.13 H+3.9510^{-2} S+0.26 R I+7.18$

where: $O C$ is percentage estimation of oil content in $F F B, \mathrm{R}$ and $B$ are the average of red and blue DN in the object, $g$ and $b$ are the average of normalized green and blue DN in the object, $H$ and $S$ are the average of hue and saturation DN in the object, and $R I$ is the ripeness index of the $F F B$ sample based on the proportion of the R, G, and B in the object (Roseleena et al., 2011). Variables $G$ and $I$ were excluded being insignificant $(\mathrm{p}>0.01)$. 
T a b l e 1. FS-MLR analysis results for modelling $F F B$ quality prediction

\begin{tabular}{cccc}
\hline Regression coefficients & Fraction & OC & FFA \\
\hline Object pixel & $-4.0810^{-5}$ & 0 & -0.084 \\
R & $84289.84510^{-7}$ & 0.083 & 0 \\
G & $69454.89210^{-7}$ & 0 & 0 \\
B & $-60253.28710^{-7}$ & $-9.8610^{-2}$ & 25.009 \\
r & 0 & -17.823 & 119.211 \\
g & $1438839.57610^{-5}$ & 51.007 & -4.146 \\
b & $-5052646.30710^{-6}$ & $-518.973 \cdot 10^{-1}$ & 0 \\
H & $11710.24410^{-7}$ & $12.6110^{-2}$ & $-5.0510^{-2}$ \\
S & 0 & $3.95510^{-2}$ & $6.48410^{-2}$ \\
I & $-90579.49810^{-7}$ & 0 & $6.510^{-5}$ \\
RI & 0 & $264.05310^{-3}$ & $-900.02910^{-2}$ \\
\hline
\end{tabular}

The FFA model is given as:

$$
\begin{aligned}
& F F A=-0.084 R+250 r+119.21 g-4.14 b-0.05 S+ \\
& 0.064 I+6.510^{-5} R I-9
\end{aligned}
$$

where: $F F A$ is percentage estimation of free fatty acids in $F F B, R$ is the average of red $\mathrm{DN}$ in the object, $r, g$, and $b$ are the average of normalized red, green, and blue DN in the object, $S$ and $I$ are the average of saturation and intensity $\mathrm{DN}$ in the object, and $R I$ is the ripeness index of the FFB sample based on the proportion of the R, G, and B in the object (Roseleena et al., 2011). In the FFA model, variables $\mathrm{G}, \mathrm{B}$ and $\mathrm{H}$ were excluded $(\mathrm{p}>0.01)$. The models results for ripeness, $O C$ and $F F A$ assessment using FS-MLR method are described in Table 1.

For the models formulated using MP-ANN method, the matrix of predictors, mass, and coefficients for each regression are presented in Tables 2 and 3, where values indicating synaptic network mass and strength of connections are given. The models developed by two statistical methods perform differently in predicting each of three assessed $F F B$ properties. For ripeness fraction and $O C$ prediction, models developed using MP-ANN statistical analysis perform better, while FS-MLR statistical analysis has better model for predicting the FFA. The comparative results of the models are presented in Table 4.

Out of three models for assessing $F F B$ properties, only the performance of $F F B$ ripeness fraction classification can be done using the receiver operating characteristic (ROC) curve analysis. Selection of ROC analysis was considered of importance for performance analysis of $F F B$ fraction classification to better understand the sensitivity and specificity balance of the models, while reducing the determination scores. The ROC performance analysis was closely correla- ted to the area under the curve, which reflected the model accuracy. The area with value near to 1 indicates that the model significantly separated the classes, while the area value of 0.50 shows that the predictor is no better than chance.

The ROC curve analysis for ripeness classification models by means of FS-MLR and MP-ANN statistical analysis method is presented in Fig. 8. The area under the curve for FS-MLR model (Fig. 9a) is 0.846 , lower compared to the value of 0.935 of MP-ANN model (Fig. 9b). This result strongly suggests that a model developed by means of MP-ANN statistical analysis for $F F B$ ripeness classification is preferable, rather than employing FS-MLR method. With $99.99 \%$ confidence interval, the area under the curve is significantly different from null hypothesis true area. Moreover, since the p-value was achieved as 0.000 , it was concluded that the classification results significantly were better than by chance.

All colour features extracted from the object image were used as input variables or predictors in generating all models. For the $O C$ and FFA models, the regressions were performed between results obtained from laboratory analysis and image features. These features, considered as predictors, were removed from calculation whenever they did not contribute significantly to the models. The prediction results of FFB $O C$ and FFA models are displayed in Figs 9 and 10, respectively.

The prediction accuracies of $F F B$ features (ripeness, $O C$ and $F F A$ ) of the models in this research provided acceptable values. The best prediction accuracy $\left(\mathrm{R}^{2}\right)$ of ripeness model is $93.5 \%$ with SEP of 0.065 . For $O C$ and $F F A$ assessments, model accurately predicts $96.41 \%$ and $89.32 \%$, respectively, while the SEP predictions are 0.044 and 0.068 for $O C$ and FFA, respectively. Compared to the manual laboratory analysis, the models deliver faster results at a fraction of costs without damaging the FFB samples. It should 
T a b l e 2. MP-ANN analysis results for modelling $F F B$ quality prediction, input layer

\begin{tabular}{|c|c|c|c|c|c|c|c|c|c|c|c|c|c|}
\hline \multirow{3}{*}{ Predictor } & & \multicolumn{12}{|c|}{ Parameter estimates } \\
\hline & & \multicolumn{12}{|c|}{ Hidden layer 1} \\
\hline & & $\mathrm{H}(1: 1)$ & $\mathrm{H}(1: 2)$ & $\mathrm{H}(1: 3)$ & $\mathrm{H}(1: 4)$ & $\mathrm{H}(1: 5)$ & $\mathrm{H}(1: 6)$ & $\mathrm{H}(1: 7)$ & $\mathrm{H}(1: 8)$ & $\mathrm{H}(1: 9)$ & $\mathrm{H}(1: 10)$ & $\mathrm{H}(1: 11)$ & $\mathrm{H}(1: 12)$ \\
\hline \multirow{12}{*}{$\begin{array}{l}\text { Input } \\
\text { layer }\end{array}$} & (Bias) & -0.405 & -0.566 & -0.506 & -0.260 & 0.250 & 0.847 & 0.796 & -0.528 & 1.218 & 0.661 & -0.862 & 0.290 \\
\hline & $\begin{array}{c}\text { Object } \\
\text { pixel }\end{array}$ & -1.012 & 0.830 & -0.602 & -1.002 & 0.258 & 2.753 & -0.279 & -0.090 & -0.161 & -0.206 & 0.419 & 0.020 \\
\hline & $\mathrm{R}$ & 0.730 & -0.976 & 1.045 & -1.068 & 0.524 & -1.879 & -0.572 & 0.191 & 0.667 & 0.530 & -0.077 & 0.498 \\
\hline & G & 0.864 & -1.195 & -0.621 & 0.640 & 0.049 & -1.398 & -0.141 & -0.393 & -0.646 & -0.027 & -0.374 & 0.130 \\
\hline & $\mathrm{B}$ & -0.847 & 1.760 & -0.314 & -0.570 & -0.331 & 1.570 & -0.096 & 0.146 & -0.182 & -0.003 & 0.415 & 0.303 \\
\hline & $\mathrm{r}$ & -0.685 & -2.081 & 0.457 & 0.047 & 0.447 & -1.090 & -1.034 & -0.440 & 0.783 & 0.112 & 0.194 & -0.250 \\
\hline & $\mathrm{g}$ & 1.259 & 0.518 & 1.115 & 0.021 & 0.571 & -1.287 & -0.044 & 0.416 & -0.089 & -0.194 & 0.039 & -0.628 \\
\hline & $\mathrm{b}$ & 0.509 & -0.545 & -1.146 & 0.921 & -1.196 & 0.731 & -0.192 & -0.742 & 0.327 & 0.567 & 0.441 & 0.492 \\
\hline & $\mathrm{H}$ & -0.354 & -2.212 & -2.136 & -0.222 & 0.288 & 1.780 & 1.197 & 0.465 & 0.441 & 0.373 & -0.474 & 0.563 \\
\hline & S & 0.200 & 0.515 & 0.139 & -0.097 & -0.586 & 2.930 & 1.553 & 0.058 & -0.123 & 0.178 & 0.466 & -0.387 \\
\hline & I & 0.126 & 0.162 & 0.857 & -0.144 & -0.612 & -0.174 & 0.749 & 0.759 & -0.224 & -0.553 & -0.066 & 0.397 \\
\hline & RI & -0.163 & 0.849 & -0.297 & 0.665 & 0.211 & -1.301 & -0.241 & 0.059 & 0.033 & -0.237 & -0.175 & -0.310 \\
\hline
\end{tabular}

T a b l e 3. MP-ANN analysis results for modelling $F F B$ quality prediction, output layer

\begin{tabular}{ccccccccccc}
\hline & & \multicolumn{7}{c}{ Parameter estimates } \\
\cline { 3 - 11 } Predictor & & \multicolumn{7}{c}{ Output layer } \\
\cline { 3 - 11 } & & F0 & F1 & F2 & F3 & F4 & F5 & F6 & OC & FFA \\
\hline Hidden & (Bias) & 0.582 & -0.189 & 0.736 & -0.289 & 0.222 & -0.251 & 0.233 & -0.520 & 0.323 \\
layer 1 & $\mathrm{H}(1: 1)$ & 0.921 & -1.488 & 0.282 & -0.159 & 0.235 & -0.055 & 0.102 & -0.232 & -0.263 \\
& $\mathrm{H}(1: 2)$ & 0.707 & -0.929 & 0.415 & -0.069 & -0.056 & 0.060 & -0.326 & -0.035 & -3.681 \\
& $\mathrm{H}(1: 3)$ & 0.703 & -0.109 & -1.117 & 0.676 & -0.279 & 0.058 & -0.200 & -0.076 & -2.555 \\
& $\mathrm{H}(1: 4)$ & 0.258 & -0.418 & 0.336 & -0.036 & -0.485 & 0.105 & 0.291 & -0.366 & 1.989 \\
& $\mathrm{H}(1: 5)$ & -0.797 & -0.274 & 0.719 & -0.025 & 0.720 & 0.012 & -0.038 & 1.316 & 0.863 \\
& $\mathrm{H}(1: 6)$ & 0.446 & -0.639 & -0.040 & -0.012 & 0.408 & 0.001 & -0.208 & 0.007 & -3.753 \\
& $\mathrm{H}(1: 7)$ & 0.673 & 0.261 & -0.647 & -0.374 & 0.043 & 0.143 & -0.317 & 1.614 & -2.273 \\
& $\mathrm{H}(1: 8)$ & -0.630 & 0.460 & 0.668 & 0.137 & -0.960 & 0.033 & 0.272 & -0.354 & 0.710 \\
& $\mathrm{H}(1: 9)$ & -0.389 & -0.009 & -0.159 & 0.366 & -0.819 & 0.792 & 0.044 & -0.252 & 0.479 \\
& $\mathrm{H}(1: 10)$ & -0.365 & 0.334 & -0.512 & 0.926 & 0.339 & -0.838 & 0.142 & 0.403 & 0.348 \\
& $\mathrm{H}(1: 11)$ & 0.213 & -0.419 & 0.111 & -0.203 & 0.276 & -0.034 & 0.105 & 1.261 & 1.005 \\
& $\mathrm{H}(1: 12)$ & 0.419 & -0.356 & 0.435 & 0.112 & -0.689 & 0.330 & -0.221 & 0.224 & 0.120 \\
\hline
\end{tabular}

T a b l e 4. Performance comparison of models for $F F B$ quality determination

\begin{tabular}{ccccccccc}
\hline Model & \multicolumn{3}{c}{ FS-MLR Method } & \multicolumn{3}{c}{ MP-ANN Method } \\
\hline & $\begin{array}{c}\text { Calibration } \\
\left(\mathrm{R}^{2}\right)\end{array}$ & SEC & $\begin{array}{c}\text { Validation } \\
\left(\mathrm{R}^{2}\right)\end{array}$ & SEP & $\begin{array}{c}\text { Calibration } \\
\left(\mathrm{R}^{2}\right)\end{array}$ & SEC & $\begin{array}{c}\text { Validation } \\
\left(\mathrm{R}^{2}\right)\end{array}$ & SEP \\
\hline Ripeness & 0.844 & 0.305 & 0.846 & 0.36 & 0.974 & 0.03 & 0.935 & 0.065 \\
OC & 0.945 & 0.381 & 0.938 & 0.456 & 0.969 & 0.018 & 0.964 & 0.044 \\
$F F A$ & 0.896 & 0.067 & 0.893 & 0.068 & 0.78 & 0.168 & 0.645 & 0.317 \\
\hline
\end{tabular}


a

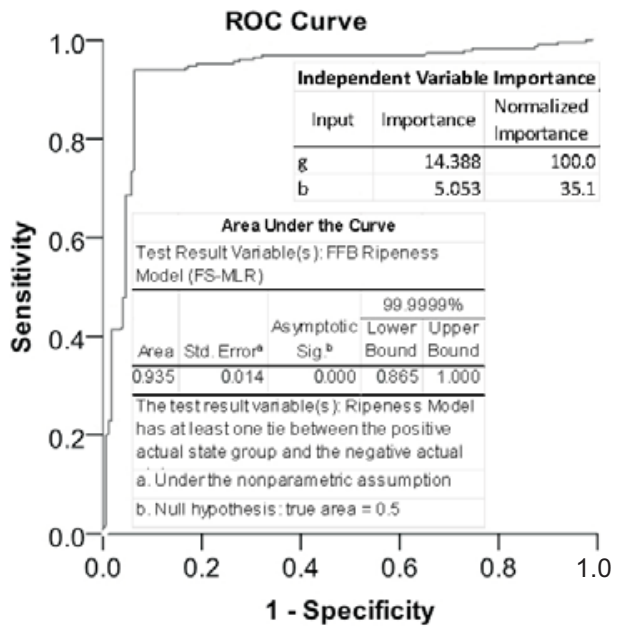

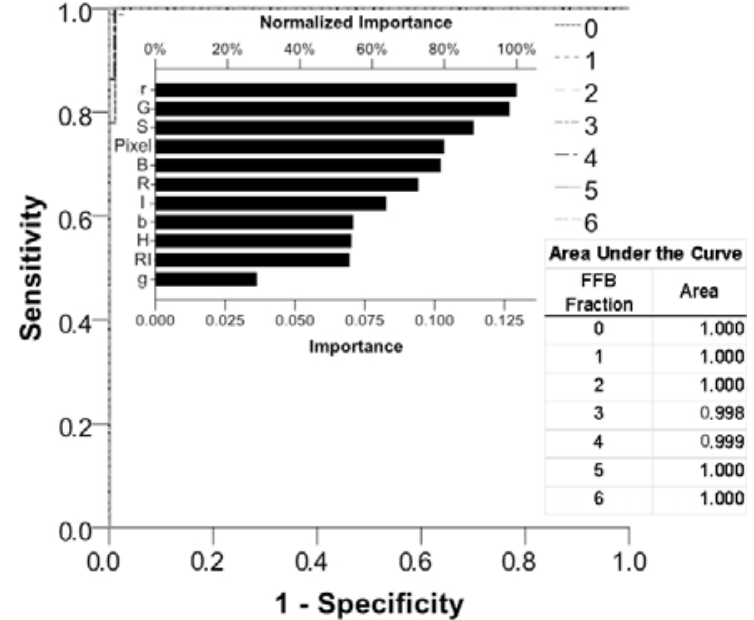

Fig. 8. ROC analysis for ripeness fraction model using: a - FS-MLR, and b-MP-ANN.

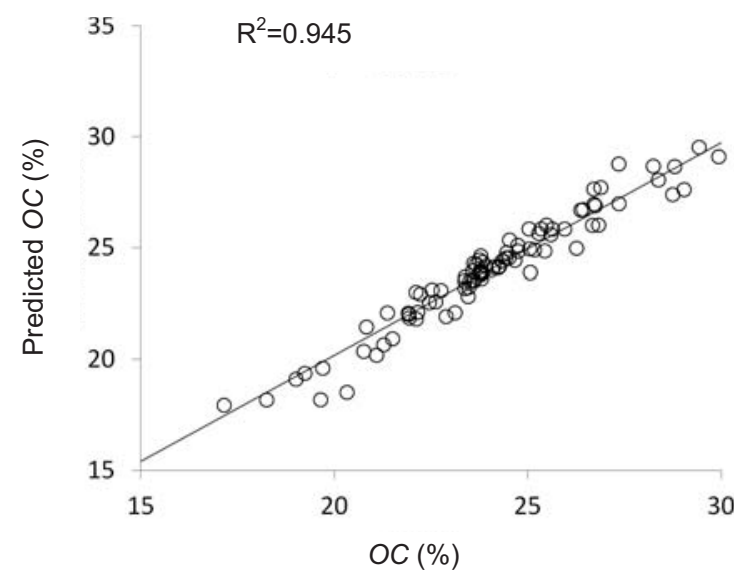

$\mathrm{b}$

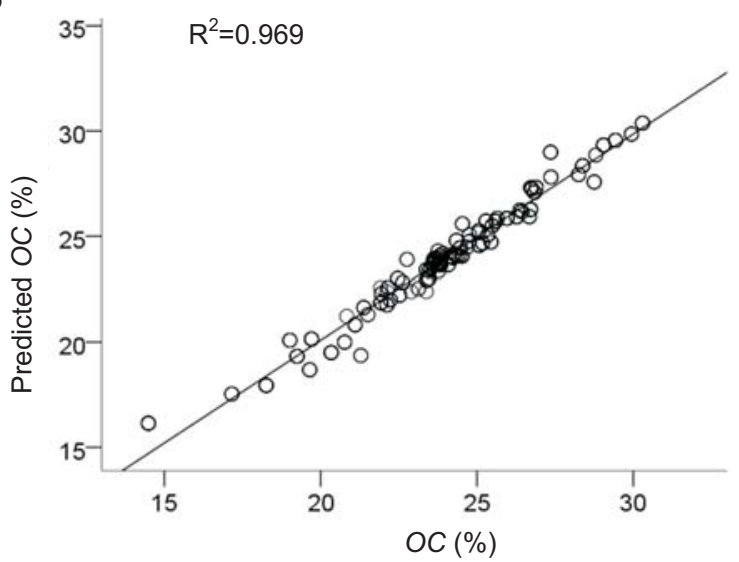

Fig. 9. FFB $O C$ model validation using: $\mathrm{a}-\mathrm{FS}-\mathrm{MLR}$, and $\mathrm{b}-\mathrm{MP}-\mathrm{ANN}$.

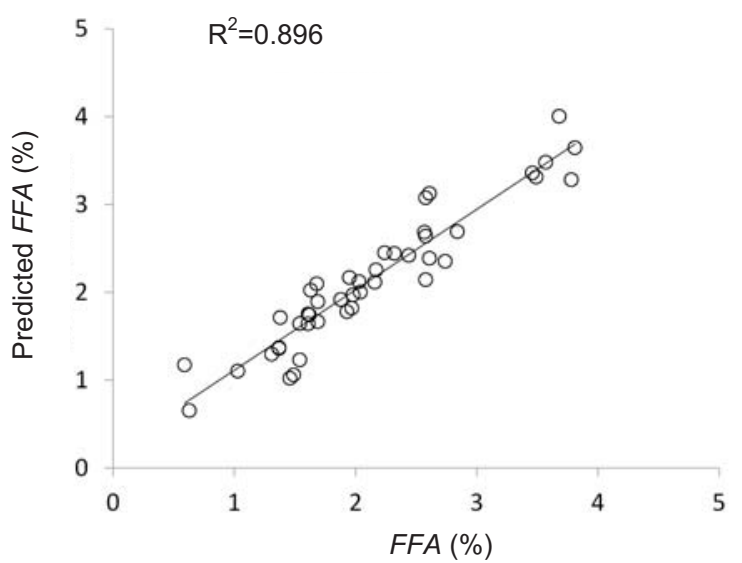

b

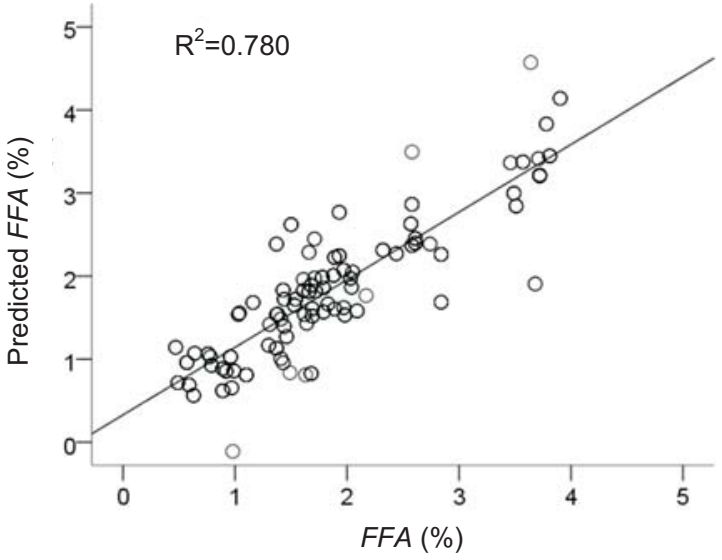

Fig. 10. FFB FFA model validation using: a - FS-MLR, and b-MP-ANN. 
be noted that the laboratory analyses were prone to error due to human factors, thus the results cannot be guaranteed $100 \%$ error free.

The novelty of this research is the machine vision inspection system that had been further developed to be able to assess three $F F B$ properties, namely ripeness fraction, oil content and free fatty acid level, using models developed by means of two statistical analysis: FS-MLR and MP-ANN methods. The system is more compact and the algorithm in the image processing program is simplified, resulting in reduction of time required for estimating the $F F B$ s ripeness, $O C$ and $F F A$, as well as the whole grading time. Compared to other available models (Shariff et al., 2004; Junkwon et al., 2009; Razali et al., 2011) and our previous work (Makky and Soni, 2013b), the developed machine gives better accuracy in validating $F F B$ s ripeness, $O C$ and $F F A$. Moreover, this automatic grading machine was tested using more samples of $F F B S$ and directly examines the whole bunch of $F F B$, therefore eliminating the need of taking samples of individual fruitlets. Hence, the machine provides faster, more practical and non-destructive examination results. Application of machine vision in this machine ensures the consistency of inspection results. The works also provides a new approach to non-destructive analysis for oil palm $F F B$ while providing a system that might be useful in the agricultural sector or other sectors that require it.

In the tests, the placement of FFBs on the conveyor belt was not regulated, resulting in different bunch orientations. However, this showed no influence on the accuracy. The FFBs were fed by the operator onto machine, with its handle facing toward the inspection chamber. Motor and transmission combination arrangement produced a constant belt speed of $110 \mathrm{~mm} \mathrm{~s}^{-1}$, with the software processing the image less than $5 \mathrm{~s}$ for examination of each $F F B$. The machine examination capacity is more than $12 \mathrm{t}$ of $F F B$ s per hour; which fairly satisfies mill grading capacity requirement. All $F F B$ samples used in the test showed no major bruise, and hence the grading process can be considered safe in handling the bunch without damaging it. This work opens an opportunity to further develop the automatic grading systems to be applied in agricultural sectors as well as in other sectors. More methods can be developed to increase the efficiency and effectiveness of this kind of system.

\section{CONCLUSIONS}

1. In this research, an automatic inspection machine for examining ripeness fraction, oil content and free fatty acid of oil palm fresh fruits bunch was developed and tested through a series of field tests. It is able to automatically examine 12 t of fresh fruit bunches per hour without causing injuries to the bunch.

2. Chemical analysis conducted in the laboratory showed that moderately ripe fresh fruit bunches in the fraction range from 1 to 3 correspond to acceptable quality, as compared to fresh fruit bunches in higher fractions, based on their oil content and free fatty acid.

3. For the prediction of ripeness and oil content of fresh fruit bunch, the best models were created using the multilayer-perceptron artificial neural network method, while for the free fatty acid assessment the best model was obtained by means of forward stepwise multiple linear regression. The best prediction accuracy of ripeness model is $93.5 \%$ with 0.065 standard error of prediction. For oil content and free fatty acid assessments, model accurately predicts 96.41 and $89.32 \%$, respectively, while the standard errors of prediction are 0.044 and 0.068 for oil content and free fatty acid, respectively.

\section{REFERENCES}

AOCS, 2004. Official methods and recommended practices of the American Oil Chemists Society. Sampling and analysis of commercial fats and oil. American Oil Chemists Society, Urbana, IL, USA.

APHA, 2005. Standard Methods for the Examination of Water and Wastewater, APHA, AWWA, WEF, part 5220 B. American Public Health Association, Washington DC, USA.

Arefi A., Motlagh A.M., and Teimourlou R.F., 2011. Wheat class identification using computer vision system and artificial neural networks. Int. Agrophys., 25, 319-325.

Arenas-Ocampo M., Alamilla-Beltrán L., Vanegas-Espinoza P., Camacho-Díaz B., Campos-Mendiola R., Gutiérrez-López G., and Jiménez-Aparicio A., 2012. Fractal morphology of Beta vulgaris L. cell suspension culture permeabilized with Triton X-100. Int. Agrophys., 26, 1-6.

Asadi V., Raoufat M., and Nassiri S., 2012. Fresh egg mass estimation using machine vision technique. Int. Agrophys., 26, 229-234.

Bobbio P.A., and Bobbio F.O., 2001. Chemical Food Processing (in Portuguese). Varela, Sao Paulo, Brazil.

Briseno-Tepepa B.R., Jiménez-Peréz J.L., Saavedra R., GonzálezBallesteros R., and Suaste E., Cruz-Orea A., 2008. Photopyroelectric microscopy of plant leaves. Int. J. Thermophysics, 29(6), 2200-2205.

Gonzalez R.C. and Woods R.E., 2008. Digital Image Processing. Pearson Prentice Hall, Pearson Education, Inc. New Jersey, USA.

Gonzalez-Ballesteros R., Gonzalez M.C.O., Suaste-Gomez E., and Cruz-Orea A., 2006. Polivinilidene fluoride (PVDF) applied to photopyroelectric microscopy. Proc. 3rd Int. Conf. Electrical and Electronics Engineering, September 6-8, Veracruz, Mexico.

Hadi S., Ahmad D., and Akande F.B., 2009. Determination of the bruise indexes of oil palm fruits. J. Food Eng., 95, 322-326.

Hernandez A.C., Cruz O.A., Ivanov R., Dominguez P.A., Carballo C.A., and Moreno I., 2011. Optical properties of maize seeds. Int. Agrophys., 25, 223-227.

Hernandez A.C., Dominguez P.A., Cruz O.A., Ivanov R., Carballo C.A., and Zepeda B.R., 2010. Laser in agriculture. Int. Agrophys., 24, 407-422. 
IOPRI, 1997. Palm oil and palm oil mill waste management. Team of standardization for palm oil processing. Indonesian Oil Palm Research Institute (IOPRI) (in Indonesian). Directorate general of forestry, Indonesia.

Jaffar A., Jaafar R., Jamil N., Low C. Y., and Abdullah B., 2009. Photogrammetric grading of oil palm fresh fruit bunches. Int. J. Mechanical Mechatronics Eng., 9(10), 7-13.

Jamil N., Mohamed A., and Abdullah S., 2009. Automated grading of palm oil fresh fruit bunches (FFB) using neuro-fuzzy technique. Int. Conf. Soft Computing and Pattern Recognition, 245-249.

Junkwon P., Takigawa T., Okamoto H., Hasegawa H., Koike M., Sakai K., Siruntawineti J., Chaeychomsri W., Vanavichit A., Tittinuchanon P., and Bahalayodhin B., 2009. Hyperspectral imaging for nondestructive determination of internal qualities for oil palm (ElaeisguineensisJacq. var. tenera). Agric. Information Res., 18(3), 130-141.

Koenderink N.J.J.P., Broekstra J., and Top J.L., 2010. Bounded transparency for automated inspection in agriculture. Computers Electronics Agric., 72, 27-36.

Kondo N., 2010. Automation on fruit and vegetable grading system and food traceability. Trends in Food Sci. Technol., $21,145-152$.

Liu Y., Chen X., and Ouyang A., 2008. Nondestructive determination of pear internal quality indices by visible and nearinfrared spectrometry. LWT - Food Sci. Technol., 41, $1720-1725$

Makky M., Herodian S., and Subrata I.D.M., 2004. Design and Technical test of visual sensing system for palm oil harvesting robot. Proc. Int. Seminar on Advanced Agric. Eng. Farm Work Operation, August 25-26, Bogor, Indonesia.

Makky M. and Soni P., 2013a. Towards sustainable green production: exploring automated grading for oil palm fresh fruit bunches (FFB) using machine vision and spectral analysis. Int. J. Advanced Sci. Eng. Information Technol., 3(1), 1-7.

Makky M. and Soni P., 2013b. Development of an automatic grading machine for oil palm fresh fruit bunches (FFBs) based on machine vision. Computers Electronics Agric., 93, 129-139.

Makky M. and Soni P., 2014. In situ quality assessment of intact oil palm fresh fruit bunches using rapid portable non-contact and non-destructive approach. J. Food Eng., 120, 248-259.

Matsushima U., Graf W., Zabler S., Manke I., Dawson M. Choinka G., Hilger A., and Herppich W., 2013. 3D-analysis of plant microstructures: advantages and limitations of synchrotron X-ray microtomography. Int. Agrophys., 27, 23-30.

Mireei S.A., Mohtasebi S.S., Massudi R., Rafiee S., and Arabanian A.S., 2010. Feasibility of near infrared spectroscopy for analysis of date fruits. Int. Agrophys., 24, 351-356.

Naes T. and Mevik B.H., 2001. Understanding the collinearity problem in regression and discriminant analysis. J. Chemometrics, 15(4), 413-426.

Nicolai B.M., Beullens K., Bobelyn E., Peirs A., Saeys W., Theron K.I., and Lammertyn J., 2007. Nondestructive measurement of fruit and vegetable quality by means of NIR spectro- scopy: A review. Postharvest Biol. Tech., 46, 99-118.

NSAI, 2006. Crude Palm Oil. National Standardization Body of Indonesia (in Indonesian). SNI 01-2901-2006. Stipulation No. 107/KEP/BSN/05/2006, Indonesia.

Osawa C.C., Goncalves L.A.G., and Ragazzi S., 2007. Correlation between free fatty acids of vegetable oils evaluated by rapid tests and by the official method. J. Food Composition Analysis, 20, 523-528.

Razali M.H., Ismail W.I.W., Ramli A.R., Sulaiman N., and Harun M.H.B., 2011. Technique on simulation for real time oil palm fruits maturity prediction. African J. Agric. Res., 6(7), 1823-1830.

Roseleena J., Nursuriati J., Ahmed J., and Low C.Y., 2011. Assessment of palm oil fresh fruit bunches using photogrammetric grading system. Int. Food Res. J., 18(3), 999-1005.

Saad B., Ling C.W., Jab M.S., Lim B.P., Ali A.S.M., and Wai W.T., 2006. Determination of free fatty acids in palm oil samples using non-aqueous flow injection titrimetric method. Food Chemistry, 102, 1407-1414.

Shaarani S.M.D., Cardenas-Blanco A., Amin M.H.G., Soon N.G., and Hall L.D., 2010. Monitoring Development and Ripeness of Oil Palm Fruit (Elaeis guneensis) by MRI and Bulk NMR. Int. J. Agric. Biol., 12(1), 101-105.

Shariff R., Nor A., Adnan R.M., Shattri M., Rohaya H., and Roop G., 2004. Correlation between oil content and $\mathrm{dn}$ values. University Putra Malaysia, GISdevelopment.net

Siregar I.M., 1976. Assessment of ripeness and crop control in oil palm. Proc. Malaysian Int. Agric. Oil Palm Conf., June14-17, Kuala Lumpur, Malaysia.

Zapotoczny P., 2012. Application of image texture analysis for varietal classification of barley. Int. Agrophys., 26, 81-90.

Zdunek A., Adamiak A., Pieczywek P.M., and Kurenda A., 2014. The biospeckle method for the investigation of agricultural crops: A review. Optics Lasers Eng., 52, 276-285. 